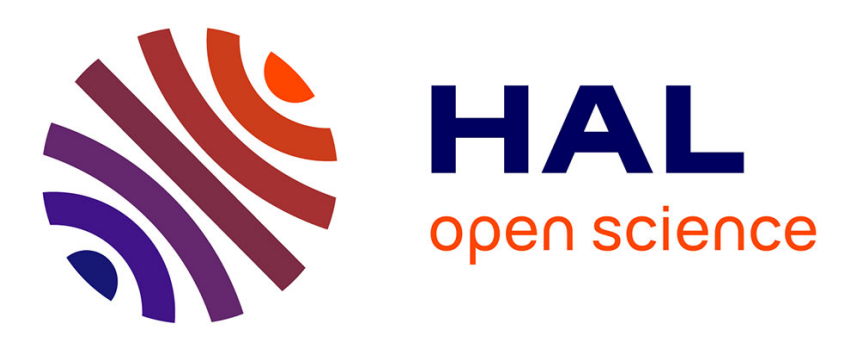

\title{
Modélisation des effets mécaniques des transformations de phases pour le calcul de structures
}

\author{
J.-Ch. Videau, Georges Cailletaud, André Pineau
}

\section{To cite this version:}

J.-Ch. Videau, Georges Cailletaud, André Pineau. Modélisation des effets mécaniques des transformations de phases pour le calcul de structures. Journal de Physique IV Proceedings, 1994, 04 (C3), pp.C3-227-C3-232. 10.1051/jp4:1994331 . jpa-00252528

\section{HAL Id: jpa-00252528 https://hal.science/jpa-00252528}

Submitted on 1 Jan 1994

HAL is a multi-disciplinary open access archive for the deposit and dissemination of scientific research documents, whether they are published or not. The documents may come from teaching and research institutions in France or abroad, or from public or private research centers.
L'archive ouverte pluridisciplinaire HAL, est destinée au dépôt et à la diffusion de documents scientifiques de niveau recherche, publiés ou non, émanant des établissements d'enseignement et de recherche français ou étrangers, des laboratoires publics ou privés. 


\title{
Modélisation des effets mécaniques des transformations de phases pour le calcul de structures
}

\author{
J.-Ch. VIDEAU, G. CAILLETAUD* et A. PINEAU*
}

Centre des Matériaux, Ecole des Mines de Paris et Direction des Constructions Aéronautiques, Ministère de la Défense, France

* Centre des Matériaux, Ecole des Mines de Paris, URA 866 du CNRS, BP. 87, 91003 Evry cedex, France

\begin{abstract}
A set of constitutive equations are proposed to take into account the predominant mechanical effects due to solid state phase transformations in steels under multi-axial loading. The aim of this work is to formalize, for structural computations (heat treatment and welding), the main experimental and theoretical results available in the literature. The internal stresses generated by the transformation induced flow are represented by internal variables in the framework of the thermodynamics of the irreversible processes. We propose a mathematical formulation of the strain-hardening effects on the transformation induced plastic flow and of the transformation induced hardening on the "classical" plasticity. The constitutive equations have been implemented into the F.E. code ZeBuLoN and we briefly present a thermo-metallurgical and mechanical simulation with hardening couplings.
\end{abstract}

\section{INTRODUCTION}

Afin de rendre compte des principaux effets mécaniques macroscopiques des transformations de phases à l'état solide dans les aciers, on propose un modèle de comportement mécanique qui permet de formaliser, pour le calcul de structures, les principaux résultats expérimentaux et théoriques dont on dispose aujourd'hui sur le sujet. On s'intéresse ici essentiellement à la modélisation mécanique multi-axiale de la plasticité induite par la transformation. Les applications pratiques visées par cette étude sont le traitement thermique et le soudage des aciers et alliages métalliques, avec pour objectifs de mieux maîtriser, par le calcul, le procédé de traitement en lui-même (problème des "tapures" de trempe par exemple), ainsi que les contraintes et déformations résiduelles engendrées par le traitement.

Nous utilisons une formulation visco-plastique du comportement qui nous permet de dériver naturellement, à partir des mécanismes sous-jacents, la forme mathématique des équations décrivant l'écoulement plastique induit par la transformation. Une attention particulière est portée à la prise en compte des contraintes internes générées par la plasticité de transformation. Ces contraintes sont en effet la cause d'importantes déformations résiduelles, souvent mal maîtrisées. Elles peuvent également exercer une influence significative sur les cinétiques de transformation $/ 1 /$. Cette description des contraintes internes repose sur la notion de variable interne de la thermodynamique des processus irréversibles, cadre général proposé par Hamata /2/ pour l'étude des couplages entre viscoplasticité et transformations de phases. Nous proposons dans ce cadre une formulation mathêmatique phénoménologique des effets de l'écrouissage dynamique sur la plasticité induite par la transformation et de l'écrouissage induit par la transformation sur la plasticité "classique".

Les modèles proposés ont été implantés dans le code de calcul de structures par éléments finis ZeBuLoN développé au Centre des Matériaux et nous présentons un exemple de calcul (thermique, métallurgique, mécanique) avec couplage entre les différents écrouissages. L'algorithme numérique utilisé pour intégrer les équations de comportement permet de "transiter", de façon continue, du comportement viscoplastique de haute température au comportement plastique indépendant du temps de basse température, ce qu'il est nécessaire de pouvoir faire pour reproduire correctement un traitement thermique. L'utilisation que l'on peut actuellement faire de tels modèles est cependant "minimale", dans la mesure où, sí l'on connaît assez bien les mécanismes physiques en jeu, on ne dispose pas, à l'heure actuelle, d'une base d'essais mécaniques suffisante. Certains essais de caractérisation sont actuellement en cours au Centre des Matériaux et feront l'objet d'une publication ultérieure.

\section{BASES PHYSIQUES DE LA MODELISATION}

D'un point de vue phénoménologique, on appelle "plasticité de transformation" l'écoulement plastique "anormal" provoqué par l'application d'une contrainte sur un échantillon en cours de transformation. On constate en effet expérimentalement qu'une éprouvette en acier, de structure austénitique par exemple, présente, après avoir été trempée sous charge, une déformation résiduelle importante, en plus du simple changement de volume associé à la transformation de phases austénite-martensite induite par la 
trempe. Si l'on répète de nombreuses fois le processus (trempe-austénitisation-trempe... sous charge), les allongements obtenus peuvent être considérables.

\section{Principaux résultats expérimentaux}

Il semble acquis que, sous faible contrainte uniaxiale, la déformation créée par une transformation complète soit sensiblement proportionnelle à la contrainte appliquée :

$$
\varepsilon^{\mathrm{pt}}=\mathrm{K} \sigma
$$

$\mathrm{K}$ est une constante de l'ordre de $10^{-4} \mathrm{MPa}^{-1}$.

Le cas des transformations incomplètes est plus mal connu, aucune loi entièrement satisfaisante n'ayant pu être mise en évidence. Il est cependant établi que la plasticité de transformation n'est fonction que de la fraction transformée, mais ne dépend pas de la vitesse de transformation $/ 3 / . \mathrm{La}$ plupart des auteurs introduisent donc un facteur d'échelle $f(z)$, fonction de la fraction transformée $z$, valant 0 pour $z=0$ et 1 pour $z=1$.

La forme la plus simple et la plus souvent utilisée dans le cas des transformations martensitiques est la suivante $/ 4$ 6/:

$$
\varepsilon^{\mathrm{pt}}=\mathrm{K} \sigma \mathrm{f}(\mathrm{z}) \quad \text { avec } \mathrm{f}(\mathrm{z})=(2-\mathrm{z}) \mathrm{z}
$$

Cette formule rend compte de l'observation expérimentale selon laquelle le taux d'écoulement plastique est important en début de transformation et tend vers 0 en fin de transformation de phases.

Dans le cas des transformations avec diffusion, on utilisera un facteur d'échelle linéaire $/ 7 /$ :

$$
f(z)=z
$$

\section{Mécanismes de la plasticité de transformation}

Deux mécanismes de nature complètement différente sont responsables de la plasticité de transformation. Ce sont, pour résumer, les effets macroscopiques de l'interaction contrainte-produits de transformation d'une part, de l'accommodation plastique locale des incompatibilités de déformation thermo-métallurgique d'autre part.

Le premier mécanisme est bien connu dans le cas des transformations martensitiques et assimile ce type de transformation de phases à un mode de déformation très voisin du maclage. Selon cette interprétation, proposée par Scheil /8/ puis développée par Magee /9/, lapplication d'une contrainte orienterait les produits de transformation. $\mathrm{Ce}$ mécanisme est à la base des propriétés mécaniques des alliages à mémoire de forme, mais on peut également observer dans les aciers des plaquettes de martensite orientées $/ 10 / / 11 /$, ainsi qu'un effet mémoire non négligeable $/ 11 /$.

La seconde interprétation, initialement proposée par De Jong et Rathenau $/ 12 /$, raffinée par Greenwood et Johnson (GJ) $/ 13 /$, puis Leblond $/ 14 /$, considère que la plasticité de transformation est due à la polarisation par la contrainte appliquée de la micro-plasticité engendrée par les incompatibilités de volumes associées à la transformation de phases. Les contraintes générées par l'avancée du front de transformation créent, en d'autres termes, des dislocations mobiles que le champ de contraintes appliqué fait glisser. Nous réserverons l'expression "plasticité induite par la transformation" à ce seul mécanisme. On notera que, dans le cas de matériaux multiphasés stables, les différences de coefficients de dilatation thermique peuvent donner naissance à un écoulement plastique de même nature. C'est ce qu'on observe typiquement dans les composites à matrice métallique /15/.

Dans le cas des transformations martensitiques, on admet que la variation de volume (partie isotrope de la déformation de transformation) est adaptée par déformation plastique de la phase-mère (mécanisme de GJ), le cisaillement (partie déviatorique) étant adapté par avancée de la transformation ou création d'autres plaquettes d'accommodation (mécanisme de Magee). Une martensite dont la dilatation de transformation est faible, ne doit, selon cette hypothèse, générer aucune plasticité de transformation de type GJ. C'est ce qu'on observe effectivement dans les martensites "thermoélastiques" des alliages à mémoire de forme. Le calcul effectué par De Jong et Rathenau /12/, en bon accord avec l'expérience, permet de quantifier cette règle et confirme l'hypothèse de Greenwood et Johnson selon laquelle seule la dilatation de transformation serait motrice.

\section{Approches théoriques micro-macro}

Greenwood et Johnson /13/ traitent le cas d'une transformation complète sous chargement uniaxial constant et obtiennent la relation (1), dans laquelle $K$ est proportionnel à la dilatation de transformation et inversement proportionnel à la limite d'élasticité $\sigma_{\min }$ de la phase la plus faible, supposée idéalement plastique (les autres hypothèses conduisant à ce résultat sont répertoriées par Abrassart /11/):

$$
K=\frac{5}{6} \frac{\Delta v}{v} \frac{1}{\sigma_{\min }}
$$

Cette formule est en bon accord avec un grand nombre de résultats expérimentaux $/ 16 /$.

Reprenant le modèle en îlots de GJ, Leblond /14/ développe une approche micro-macro semi-quantitative et obtient la formule multiaxiale et incrémentale suivante :

$$
\dot{\dot{\varepsilon}}^{\mathrm{pt}}=\mathrm{K} \underline{\underline{\sigma}}^{\mathrm{d}} \dot{\mathrm{z}} \ln \mathrm{z}
$$

$\sigma^{\mathrm{d}}$ est la partie déviatorique du tenseur des contraintes. Nous retiendrons de cette approche micro-macro la proportionnalité de la plasticité de transformation vis-à-vis de la partie déviatorique du tenseur des contraintes. (On remarquera la présence d'une singularité gênante à l'origine.)

Il n'y a que très peu de résultats théoriques concernant les transformations incomplètes. On citera pour mémoire la formule obtenue par Abrassart/11/(les valeurs d'écoulement obtenues à la limite $z=1$ sont, en effet, trois fois plus faibles que celles observées expérimentalement) :

$$
\varepsilon^{\mathrm{pt}}=\frac{1}{4} \frac{\Delta \mathrm{v}}{\mathrm{v}} \frac{1}{\sigma_{\min }} \sigma\left(3 \mathrm{z}-2 \mathrm{z}^{3 / 2}\right)
$$

On pourra se référer à Mitter /17/ pour une critique du modèle d'Abrassart.

\section{MODELISATION MECANIQUE}

On ne s'intéresse ici qu'à la modélisation du second type de mécanisme (mécanisme de Greenwood et Johnson). On admettra tout d'abord qu'il y a additivité de ce mode de déformation et de la déformation visco-plastique "classique" 18/. Cette hypothèse nous conduit à écrire, avec des notations évidentes, la déformation inélastique du matériau sous la forme suivante :

$$
\underline{\underline{\varepsilon}}^{\text {ine }}=\underline{\underline{\varepsilon}}^{\mathrm{rp}}+\underline{\underline{\varepsilon}}^{\mathrm{pt}}
$$


On notera au passage qu'il est inexact de considérer la plasticité de transformation comme le terme de déformation inélastique proportionnel à $\dot{z}$, dans la mesure où une variation de $\mathrm{z}$ modifie la limite d'élasticité du matériau et peut donc donner naissance à un écoulement plastique, même en l'absence d'incompatibilités de déformations libres locales.

\section{Seuils d'écoulement}

Les théories classiques de la (visco)plasticité introduisent une fonction de charge $\mathrm{f}^{\mathrm{v}} \mathrm{p}$, telle que $\mathrm{f}^{\mathrm{P}} \mathrm{P}=0$ soit l'équation du bord du domaine d'élasticité. Ces théories ne prennent pas en compte les déformations de ce domaine au cours du chargement et décomposent son évolution en un mouvement de translation d'ensemble (écrouissage cinématique) et/ou une expansion isotrope (écrouissage isotrope). Nous écrirons classiquement :

$$
\mathrm{f}^{\mathrm{vp}}=\left\|\underline{\sigma}^{\mathrm{d}}-\underline{\underline{X}}^{\mathrm{vp}}\right\|_{\mathrm{vp}}-\mathrm{R}^{\mathrm{vp}}
$$

$\mathrm{R}^{\mathrm{vp}}$ désigne la contrainte d'écoulement plastique du matériau et $\underline{\underline{X}}^{\mathrm{vp}}$ la contrainte interne générée par la déformation plastique.

Etant donné que le mécanisme plastique induit par la transformation est de même nature que le mécanisme "classique", nous proposons d'introduire une seconde fonction de charge $\mathrm{fp}^{\mathrm{t}}$, associée au mécanisme "pt", au sens où :

- si $\mathbf{f}^{\mathrm{r}}>0$, la contrainte est suffisante pour mettre en mouvement les dislocations générées par les différences de volume spécifique et donner naissance à un écoulement macroscopique,

- si $\mathrm{f}^{\mathrm{pt}}<0$, il n'y a pas d'écoulement induit par la transformation.

Nous supposons également que les contraintes internes générées par les deux mécanismes dissipatifs considérés viennent modifier les contraintes locales : la contrainte effectivement "vue" par le mécanisme pt n'est pas la contrainte appliquée, mais la contrainte réduite $\underline{\underline{\sigma}}-\underline{\underline{X}}^{\mathrm{pt}}$, où $\underline{\underline{X}}^{\mathrm{pt}}$ désigne la contrainte interne "écran" associée à ce mécanisme.

On peut a priori mettre $\mathrm{fpr}^{\mathrm{r}}$ sous la forme (8) où $\phi$ est une fonction de la contrainte effective, nulle à l'origine et où $R^{p t}$ s'interprète comme le seuil en contrainte du mécanisme induit :

$$
\mathrm{f}^{\mathrm{pt}}=\phi\left(\underline{\underline{\sigma}}-\underline{\underline{X}}^{\mathrm{pt}}\right)-\mathrm{R}^{\mathrm{pt}}
$$

On constate expérimentalement que $R^{\mathrm{pt}} \approx 0$.

Pour la même raison que plus haut, nous supposons que $\phi$ est une norme du déviateur de la contrainte effective de ce mécanisme, soit, pour un matériau isotrope:

$$
\phi=\left\|\underline{\sigma}^{d}-\underline{\underline{X}}^{\mathrm{pt}}\right\|_{\rho t}=\sqrt{\frac{3}{2}\left(\underline{\underline{\sigma}}^{\mathrm{d}}-\underline{\underline{X}}^{\mathrm{pt}}\right):\left(\underline{\underline{\sigma}}^{\mathrm{d}}-\underline{\underline{X}}^{\mathrm{pt}}\right)}
$$

\section{Ecoulement plastique}

Nous écrivons formellement :

$$
\dot{\underline{\varepsilon}}^{\mathrm{vp}}=\dot{\mathrm{v}} \underline{\underline{\underline{n}}}^{\mathrm{vp}} \quad \dot{\underline{\varepsilon}}^{\mathrm{pt}}=\dot{\mathrm{p}} \underline{\underline{n}}^{\mathrm{pt}}
$$

où nous notons :

- $\underline{\underline{n}}^{\mathrm{vp}}$ et $\underline{\underline{n}}^{\mathrm{pt}}$ les directions d'écoulement des deux mécanismes considérés,
- $\dot{v}$ et $\dot{\mathrm{p}}$ leurs intensités respectives.

Les variables "cumulées" $v$ et $p$, qui, en toute rigueur, ne sont pas des variables d'état, sont des indicateurs de l'histoire des déformations inélastiques au sens où :

$$
\mathrm{v}(\mathrm{t})=\int_{0}^{\mathrm{t}}\left\|\underline{\underline{\dot{\varepsilon}}}^{\mathrm{vp}}\right\|_{\mathrm{vp}} \mathrm{d} \tau \quad \mathrm{p}(\mathrm{t})=\int_{0}^{\mathrm{t}}\left\|\underline{\underline{\dot{\varepsilon}}}^{\mathrm{pt}}\right\|_{\mathrm{pt}} \mathrm{d} \tau
$$

Nous admettons, compte-tenu des mécanismes plastiques en jeu (par glissement de dislocations), les hypothèses de dissipativité normale :

$$
\underline{\underline{n}}^{\mathrm{v} \mathrm{p}}=\frac{\partial \mathrm{f}}{\partial \underline{\underline{\sigma}}}^{\mathrm{vp}} \quad \underline{\underline{n}}^{\mathrm{pt}}=\frac{\partial \mathrm{f}}{\partial \underline{\underline{\sigma}}}^{\mathrm{pt}}
$$

L'intensité de l'écoulement viscoplastique est, classiquement, donnée par la distance ${ }^{1}$ du point représentatif de l'état actuel dans l'espace des contraintes à la surface-seuil $f^{v e}=0$ :

$$
\dot{v}=\left\langle\frac{f^{v p}}{K^{v p}}\right\rangle^{n}
$$

où $\mathrm{K}^{\mathrm{pt}}$ et $\mathrm{n}$ sont deux coefficients de viscosité caractéristiques du matériau.

Nous admettons qu'il en est de même pour l'écoulement induit par la transformation :

$$
\dot{\mathrm{p}}=\mathrm{K}^{\mathrm{pt}}\left\langle\mathrm{f}^{\mathrm{pl}}\right\rangle \approx \mathrm{K}^{\mathrm{pt}}\left\|\underline{\underline{\sigma}}^{\mathrm{d}}-\underline{\underline{X}}^{\mathrm{pl}}\right\|_{\mathrm{pt}}
$$

Les résultats expérimentaux que nous avons rappelés au §II (uniaxiaux) nous indiquent alors que, pour une transformation martensitique ${ }^{2}$ :

$$
\mathrm{K}^{\mathrm{pt}}=\mathrm{K}(1-\mathrm{z}) \dot{\mathrm{z}} \text {, }
$$

formule valable dans le cas d'un alliage biphasé où $z$ désigne la fraction volumique transformée.

Nous proposons de symétriser cette relation de la façon suivante :

$$
K^{p t}=K_{12} z_{1}\left\langle\dot{z}_{2}\right\rangle+K_{21} z_{2}\left\langle\dot{z}_{1}\right\rangle
$$

Dans le cas d'un matériau à n phases, nous écrirons :

$$
\mathrm{K}^{\mathrm{pt}}=\sum_{\mathrm{i}, \mathrm{j}} \mathrm{K}_{\mathrm{ij}} \mathrm{z}_{\mathrm{i}}\left\langle\dot{\mathrm{z}}_{\mathrm{j}}\right\rangle
$$

où nous introduisons une matrice de plasticité de transformation $\left(\mathrm{K}_{\mathrm{ij}}\right), \mathrm{K}_{\mathrm{ij}}$ caractérisant la plasticité induite par la transformation $\mathrm{i} \rightarrow \mathrm{j}$. Cette matrice n'est généralement pas symétrique, puisque, comme l'ont observés Clinard et Sherby /19/ ou bien Kot et Weiss $/ 20 /$, l'écoulement induit par la transformation inverse peut être très différent de celui induit par la transformation directe.

Nous obtenons, tous calculs faits :

$\dot{\underline{\varepsilon}}^{\mathrm{pt}}=\left(\sum_{\mathrm{i}, \mathrm{j}} \mathrm{K}_{\mathrm{ij}} \mathrm{z}_{\mathrm{i}}\left\langle\dot{\mathrm{z}}_{\mathrm{j}}\right\rangle\right)\left(\underline{\underline{\sigma}}^{\mathrm{d}}-\underline{\underline{X}}^{\mathrm{pt}}\right)$

Dans le cas des transformations diffusionnelles, nous obtenons, de façon analogue :

\footnotetext{
$1_{<x>=x}$ si $x>0,0$ sinon.

${ }^{2}$ Nous admettons implicitement ici que le mécanisme "concurrent" de Magee n'est pas prépondérant. La formulation générale reste, en tout état de cause, valide.
} 


$$
\underline{\underline{\dot{\varepsilon}}}^{\mathrm{pt}}=\left(\sum_{\mathrm{i}, \mathrm{j}} \mathrm{K}_{\mathrm{ij}}\left\langle\dot{\mathrm{z}}_{\mathrm{j}}\right\rangle\right)\left(\underline{\underline{\sigma}}^{\mathrm{d}}-\underline{\underline{X}}^{\mathrm{pt}}\right)
$$

\section{Ecrouissage}

On fait l'hypothèse qu'il existe deux variables déformations, notées respectivement $\underline{\underline{\alpha}}^{\mathrm{vp}}$ et $\underline{\underline{\alpha}}^{\mathrm{pt}}$, associées aux contraintes internes $\underline{\underline{X}}^{\mathrm{vp}}$ et $\underline{\underline{X}}^{\mathrm{pt}^{\mathrm{t}}}$, et représentatives des microstructures créées par chacun des mécanismes dissipatifs. On fait, d'autre part, l'hypothèse d'une dépendance linéaire entre contraintes et déformations, et on écrira (provisoirement) :

$$
\underline{\underline{X}}^{\mathrm{vp}}=\mathrm{C}^{\mathrm{vp}} \underline{\underline{\underline{\alpha}}}^{\mathrm{vp}} \quad \underline{\underline{X}}^{\mathrm{pt}}=\mathrm{C}^{\mathrm{pl}} \underline{\underline{\alpha}}^{\mathrm{pl}}
$$

Il est clair cependant, d'un point de vue physique, qu'il faut introduire un couplage dans ces lois d'états : les dislocations créées par déformation plastique contribuent à modifier la contrainte "vue" par les dislocations mobiles créées par les incompatibilités de volume et réciproquement. On introduit donc pour rendre compte de ce phénomène une matrice d'écrouissage (symétrique en vertu du principe d'Onsager) telle que :

$$
\left(\begin{array}{l}
\underline{\underline{X}}^{\mathrm{vp}} \\
\underline{\underline{X}}^{\mathrm{pt}}
\end{array}\right)=\left(\begin{array}{cc}
\mathrm{C}^{\mathrm{vp}} & \mathrm{C} \\
\mathrm{C} & \mathrm{C}^{\mathrm{pt}}
\end{array}\right)\left(\begin{array}{l}
\underline{\underline{\alpha}}^{\mathrm{vp}} \\
\underline{\underline{\alpha}}^{\mathrm{pt}}
\end{array}\right)
$$

On s'intéressera plus loin au cas particulier où $\mathrm{C}^{\mathrm{v} \mathrm{p}}=\mathrm{C}^{\mathrm{p}}=\mathrm{C}$.

On admettra, en vertu du principe de normalité adopté plus haut, que les équations d'évolution de ces variables s'écrivent :

$$
-\underline{\underline{\alpha}}^{\mathrm{vp}}=\dot{\mathrm{v}} \frac{\partial \mathrm{f}^{\mathrm{vq}}}{\partial \underline{\underline{X}}^{\mathrm{vp}}} \quad-\underline{\underline{\dot{\alpha}}}^{\mathrm{pt}}=\dot{\mathrm{p}} \frac{\partial \mathrm{f}^{\mathrm{pt}}}{\partial \underline{\underline{X}}^{\mathrm{pt}}}
$$

Deux phénomènes de restauration doivent en plus être pris en compte si l'on veut rendre compte des observations physiques :

- la restauration dynamique classique, activée par la déformation plastique (donc proportionnelle à $\dot{v}$ ), dont en rend compte en introduisant un terme de "rappel" de la forme $\mathrm{d}^{\mathrm{v} p} \underline{\underline{X}}^{\mathrm{vp}}$,

- la régénération de l'écrouissage, partielle ou totale, lors de l'avancée du front de transformation (proportionnelle à p), que nous appellerons "régénération dynamique" et dont nous proposons de rendre compte en introduisant un terme de "rappel", de la forme $\mathrm{d}^{\mathrm{pt}} \underline{\underline{X}}^{\mathrm{pt}}$. La "régénération dynamique" correspond au fait que la transition de phases modifie très fortement les arrangements de dislocations responsables des contraintes internes (parois de dislocations ou boucles d'Orowan). Cela signifie, en d'autres termes, que la phase nouvellement formée n'est pas "consolidée" par l'écrouissage de la phase-mère $/ 21$. On notera que cette description de la régénération dynamique, purement phénoménologique, est cohérente avec celle adoptée pour l'écoulement plastique induit par la transformation. En particulier, elle ne prend pas en compte individuellement l'écrouissage de la phase-mère et celui de la phase-fille comme l'ont proposé d'autres auteurs $/ 14 / 21 /$, pourtant dans le cadre de modeles "unifiés" (au sens où ils ne décrivent pas les déformations plastiques individuelles de ces phases).
En conclusion, nous proposons les équations d'écrouissage :

$$
\begin{gathered}
\underline{\underline{\dot{\alpha}}}^{\mathrm{vp}}=\dot{\mathrm{v}}\left(\underline{\underline{n}}^{\mathrm{vp}}-\mathrm{d}^{\mathrm{vp}} \underline{\underline{X}}^{\mathrm{vp}}\right) \\
\dot{\underline{\alpha}}^{\mathrm{pt}}=\dot{\mathrm{p}}\left(\underline{\underline{n}}^{\mathrm{pt}}-\mathrm{d}^{\mathrm{pl}} \underline{\underline{\underline{X}}}^{\mathrm{pt}}\right)
\end{gathered}
$$

On suppose enfin qu'il existe deux variables déformations $r^{v p}$ et $r^{p t}$ respectivement associées aux contraintes d'écoulement $\mathrm{R}^{\mathrm{vp}}$ et $\mathrm{R}^{\mathrm{pt}}$ (seuil de désancrage-propagation des dislocations), Pour les mêmes raisons que celles présentées dans le cas de l'écrouissage cinématique, nous faisons les hypothèses suivantes:

- lois d'état linaires avec couplage,

- dissipation normale avec restauration et régénération dynamiques.

On écrira donc :

$$
\begin{aligned}
& \left(\begin{array}{l}
\mathrm{R}^{\mathrm{vp}} \\
\mathrm{R}^{\mathrm{pl}}
\end{array}\right)=\left(\begin{array}{c}
\mathrm{R}_{0}^{\mathrm{vp}} \\
0
\end{array}\right)\left(\begin{array}{cc}
\mathrm{q}^{\mathrm{vp}} & \mathrm{q} \\
\mathrm{q} & \mathrm{q}^{\mathrm{pt}}
\end{array}\right)\left(\begin{array}{l}
\mathrm{r}^{\mathrm{vp}} \\
\mathrm{r}^{\mathrm{pl}}
\end{array}\right) \\
& \left\{\begin{array}{l}
\dot{\mathrm{r}}^{\mathrm{vp}}=\dot{\mathrm{v}}\left(1-\mathrm{b}^{\mathrm{vp}}\left(\mathrm{R}^{\mathrm{vp}}-\mathrm{R}_{0}^{\mathrm{vp}}\right)\right) \\
\dot{\mathrm{r}}^{\mathrm{pt}}=\dot{\mathrm{p}}\left(1-\mathrm{b}^{\mathrm{pt}} \mathrm{R}^{\mathrm{pt}}\right)
\end{array}\right.
\end{aligned}
$$

\section{Autres équations du modèle}

- Tous les coefficients matériaux intervenant dans les lois d'état et d'évolution (exceptée bien sûr la matrice de plasticité de transformation) sont supposés vérifier une loi de mélange du type :

$$
\mathrm{D}\left(\mathrm{z}_{\mathrm{i}}, \mathrm{T}\right)=\sum_{\mathrm{i}} \mathrm{z}_{\mathrm{i}} \mathrm{D}_{\mathrm{i}}(\mathrm{T})
$$

où $\mathrm{D}_{\mathrm{i}}$ est le coefficient matériau de la phase i pure.

En effet, si, pour un acier donné, la structure cristalline (austénitique, perlitique, ferritique, bainitique ou martensitique) affecte peu les coefficients d'élasticité, elle est un facteur déterminant des caractéristiques de "résistance" (limite d'élasticité, modules d'écrouissage...). Il est donc important d'introduire dans le modèle une dépendance de ces caractéristiques vis-à-vis de la composition du métal. (Certains auteurs utilisent une loi de mélange plus complexe pour la limite d'élasticité /14/.)

- Soit $\varepsilon^{\text {thm }}$ la déformation linéaire que l'on observe macroscopiquement en l'absence de contrainte. Elle s'écrit (pour un matériau sans texture):

$$
\varepsilon^{\mathrm{uhm}}=\frac{1}{3 \mathrm{~V}^{0}}\left(\sum_{\mathrm{i}} \mathrm{z}_{\mathrm{i}} \mathrm{V}_{\mathrm{i}}-\sum_{\mathrm{i}} \mathrm{z}_{\mathrm{i}}^{0} \mathrm{~V}_{\mathrm{i}}^{0}\right)
$$

où $\mathrm{V}_{\mathrm{i}}$ désigne le volume spécifique de la phase $\mathrm{i}$ à la température $T$ (les exposants font référence à la configuration initiale).

Comme indiqué plus haut, nous ne prenons pas en compte, dans le modèle que nous présentons ici, l'effet de la contrainte sur les produits de transformation (mécanisme de Magee dans le cas des transformations martensitiques). Cela signifie, en d'autres termes, que nous considérons que l'équation (25) reste applicable en présence d'un champ de contraintes.

Nous écrirons donc:

$$
\underline{\underline{\varepsilon}}=\underline{\underline{\varepsilon}}^{\mathrm{e}}+\varepsilon^{\mathrm{ihm}} \underline{\underline{1}}+\underline{\underline{\varepsilon}}^{\text {ine }}
$$

Si l'on souhaite, pour des raisons pratiques, distinguer contribution "thermique" et contribution 
"métallurgique" on écrira (dans le cas où température de référence et température initiale sont égales) :

$$
\varepsilon^{\text {thm }}=\sum_{i}\left(z_{i}-z_{i}^{0}\right) \frac{V_{i}^{0}}{3 V^{0}}+\left(\sum_{i} z_{i} \alpha_{i}\right)\left(T-T^{0}\right)
$$

où $\alpha_{i}$ désigne le coefficient de dilatation thermique de la phase $\mathrm{i}$ à la température $\mathrm{T}$.

\section{DISCUSSION ET PERSPECTIVES}

Le modèle présenté rend, entre autres, compte du fait que, pour des contraintes inférieures au seuil d'écoulement plastique, l'avancée de la transformation de phases provoque un écrouissage du matériau, au sens où le seuil d'écoulement et la contrainte cinématique sont modifiées. C'est un écrouissage de type "latent".

On constate inversement, et conformément au raisonnement physique, qu'une pré-déformation plastique peut atténuer l'intensité de l'écoulement induit. L'auto écrouissage induit par la transformation peut également avoir ("dynamiquement") les mêmes conséquences (dès que $\underline{\underline{X}}^{\mathrm{pt}}$, ou même $\mathrm{R}$, deviennent non négligeables devant la contrainte appliquée). Cette propriété correspond physiquement au fait que les dislocations créées localement par les incompatibilités de volume sont moins mobiles et peut expliquer les difficultés rencontrées par les expérimentateurs pour déterminer le facteur d'échelle $\mathrm{f}(\mathrm{z})$.

On notera également:

1) que l'Eq.18 respecte le caractère isochore de la plasticité de transformation,

2) que c'est la contrainte effective, et non la contrainte nominale qui donne la direction d'écoulement. Si cette hypothèse est, physiquement, peu sujette à caution, elle n'a, à notre connaissance, pas été testée expérimentalement. Des essais de caractérisation sont en cours au Centre des Matériaux.

Il est ici clair qu'il est nécessaire, pour étudier de tels phénomènes couplés, de disposer de méthodes numériques d'identification efficaces, celles-ci ne pouvant être mises en oeuvre qu'à partir de modèles tels que celui que nous proposons. Les résultats expérimentaux dont on dispose à ce jour sont malheureusement peu nombreux.

Nous avons jusqu'à présent considéré le coefficient de plasticité de transformation $\mathrm{K}$-associé à une transformation donnée- - comme une constante du modèle. Cela revient, si l'on se reporte à la formule de GJ, à négliger d'une part les effets d'écrouissage ( $\sigma_{\mathrm{min}}$ constant) lors de la phase de "germination" (création des dislocations par "emboutissage" de la phase-mère), d'autre part la dépendance en température du changement de volume et des autres caractéristiques mécaniques des phases. Si l'on peut espérer lever cette deuxième limitation en mesurant la dépendance en température de $K$, il est certainement plus difficile de tenir compte des effets d'écrouissage sur la "germination". On peut, si nécessaire, envisager de les prendre en compte, en introduisant une variable d'état scalaire $k$ associée à la contrainte $1 / \mathrm{K}$.

\section{IMPLANTATION NUMERIQUE}

Nous avons implanté ce modèle général dans le code de calcul de structures par éléments finis ZeBuLoN (développé au Centre des Matériaux). La formulation que nous avons présentée, en termes de variables d'ćtat thermodynamiques, permet de structurer les équations sous une forme concise, adaptée au calcul numérique et permet également de rendre compte au moyen du même formalisme du comportement d'une large classe de matériaux (critère de Mises, Hill..., écrouissage cinématique, isotrope, couplages mutuels...).

Les équations d'évolution des variables internes du modèle ont été discrétisées au moyen d'une règle du point-milieu généralisée. Cette méthode possède en effet de bonnes propriétés de stabilité et de convergence. Elle conduit à un système non-linéaire que nous résolvons itérativement par méthode de Newton-Raphson. Nous avons cependant sensiblement modifié cet algorithme afin de pouvoir traiter la plasticité indépendante du temps comme cas limite de la visco-plasticité (l'idée sous-jacente a été de conserver l'équation d'évolution de la pseudo-variable d'état $v$, celle-ci "dégénérant" en "condition de cohérence").

Les figures 1 et 2 présentent les résultats de calcul d'une "trempe à induction" sur éprouvette entaillée axisymétrique en acier ferritique ( $\emptyset$ fût $22 \mathrm{~mm}, \emptyset$ section minimale $16 \mathrm{~mm}$, rayon d'entaille $5 \mathrm{~mm}$ ). Ce traitement thermique consiste à chauffer violemment par induction la couche superficielle de l'entaille de l'éprouvette, de manière à porter cette couche à la température d'austénitisation, puis à refroidir énergiquement par trempe à l'eau, afin d'obtenir, en surface, une structure martensitique plus dure, en même temps qu'une zone de contraintes de compression, favorable au comportement en service de la pièce (en particulier sa tenue en fatigue).

Nous obtenons, après traitement, une couche de martensite d'épaisseur $2,6 \mathrm{~mm}$ dans la section minimale, ainsi qu'une zone de compression d'épaisseur $3,2 \mathrm{~mm}$ environ.

Nous négligeons, dans le calcul que nous présentons, l'énergie de déformation mécanique (compte-tenu de l'importance des transferts thermiques), ainsi que les effets de la contrainte sur les cinétiques de transformation. Il est donc possible, moyennant ces hypothèses, de calculer l'évolution de la température et de la composition métallurgique indépendamment du comportement mécanique. Ce calcul préalable a été effectué sans tenir compte explicitement des effets des chaleurs latentes de transformations de phases. Les coefficients inconnus du problème thermique (taux de production de chaleur par induction dans la couche superficielle au cours du chauffage et coefficient d'échange métal-liquide) ont été identifiés ${ }^{3}$ à partir des mesures fournies par cinq thermocouples implantés dans la section médiane de l'éprouvette. Les cinétiques de transformation anisothermes (ferrite-austénite au chauffage, austénite-martensite au refroidissement) sont explicites (cinétique de Kö̈stinen pour la transformation martensitique, fraction d'austénite fonction linéaire de la température pour la transformation au chauffage). La plasticité de transformation a été négligée pour la transformation au chauffage. Le coefficient de plasticité de transformation austénite - martensite utilisé pour le calcul est de $0,5 \cdot 10^{-4} \mathrm{MPa}^{-1}$. Nous avons, pour les deux mécanismes, négligé l'écrouissage isotrope et nous avons pris pour norme caractéristique de chaque mécanisme la norme de von Mises. Nous avons négligé également les termes de "rappel" des équations d'écrouissage et nous avons, en l'absence de plus d'information, dégénéré la matrice de couplage cinématique en un simple scalaire $\mathrm{C}^{\mathrm{V} P}=\mathrm{C}^{\mathrm{P}}=\mathrm{C}$. Cela signifie que la même contrainte interne $\underline{\underline{\mathrm{X}}}$ vient, pour chacun des mécanismes, se retrancher à la

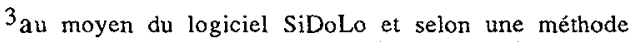
maintenant classique au Centre des Matériaux /22/. 
contrainte nominale, de telle sorte que l'écoulement "classique" et l'écoulement induit par la transformation se font suivant la (même) direction donnée par la contrainte effective $\underline{\sigma}^{d}-\underline{X}$. On obtient là, comme cas particulier, le modèle proposé et discuté par Inoue /23/.

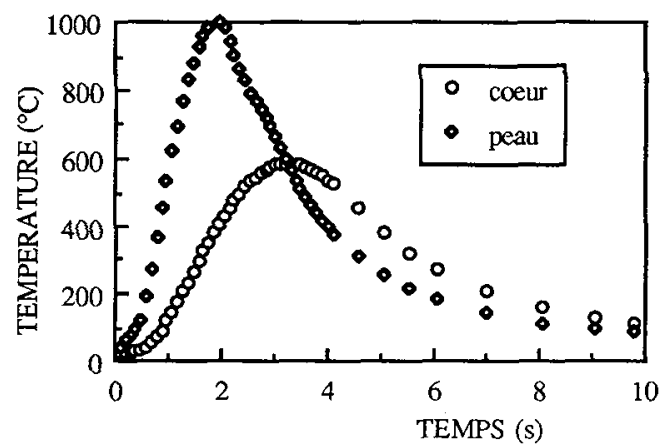

Fig.1 Evolutions de température (section médiane)

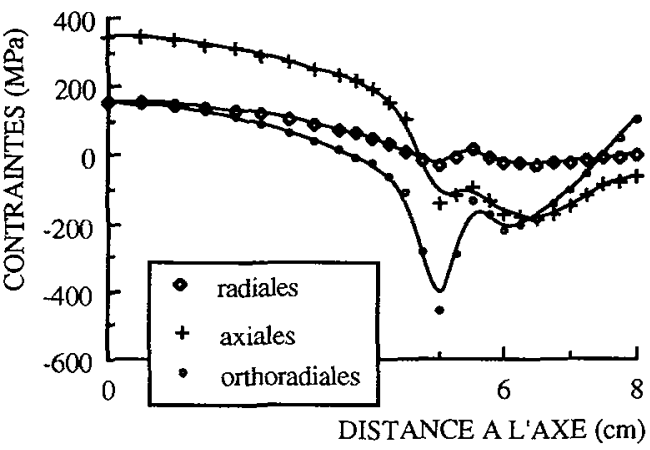

Fig.2 Contraintes résiduelles (section médiane)

\section{REFERENCES}

/1/ DENIS S., GAUTIER E., SJÖSTRÖM S. and SIMON A., 1987, "Influence of Stresses on the Kinetics of Pearlitic Transformation during Continous Cooling.", Acta Met., $35,7,1621-1632$

12/ HAMATA N., 1992, "Modélisation du couplage entre l'Élasto-viscoplasticité anisotherme et la transformation de phase d'une fonte G.S. ferritique." Thèse de doctorat Université Paris VI

/3/ DELORME J.F., 1971, "Manifestations anélastiques et transformations martensitiques.", Thèse Université de Lyon 14/ DESALOS Y., 1981, "Comportement dilatométrique et mécanique de l'austénite métastable d'un acier A533.", Rapport Irsid 95349401

15/ DENIS S., GAUTIER E., SIMON A., BECK G., 1984, "Stress Phase Transformation Interactions: Basic Principles; Modelization and Their Role in the Calculation of Internal Stresses.", Int. Symp. on the Calculation of Internal Stresses in Heat Treatment of Metallic Materials, Linkoping, Sweden, 1, 157-190

/6/ LEBLOND J.-B., MOTTET G., DEVAUX J., DEVAUX J.-C., 1984, "Mathematical Models for Description of Anisothermal Phase Transformations in Steel and Their Consequences on the Plastic Behaviour.", Int. Symp. on the Calculation of Internal Stresses in Heat Treatment of Metallic Materials, Linkoping, Sweden, 1, 191-220

/7/ GAUTIER E., SIMON A. et BECK G., 1987, "Plasticité de transformation durant la transformation perlitique d'un acier eutectoïde.", Acta Met., Vol.35/6, p.1367

/8/ SCHEIL E, 1932, Zeit. Anorg. Allgem. Chem., 207, 21

19/ MAGEE C.L., 1966, PhD Thesis, Carnegie Mellon University

/10/ MACHLIN E.S., WEINIG S., 1953, Acta Met., 1, 480

/11/ ABRASSART F., 1972, "Influence des transformations martensitiques sur les propriétés mécaniques des alliages du système $\mathrm{Fe}-\mathrm{Ni}-\mathrm{Cr}-\mathrm{C} . "$, Thèse Université de Nancy 1

/12/ DE JONG M., RATHENAU G.W., 1961, Acta Met., 9,714

/13/ GREENWOOD G.W., JOHNSON R.H., 1965, "The deformation of metals under small stresses during phase transformations.", Proc. Roy. Soc. London, A283,403-422 14/ LEBLOND J .-B ., 1989, "Mathematical modelling of transformation plasticity in steels.", Int. J. Plast., Vol.5, p.573

/15/ LeFLOUR J.C., LOCICERO R., 1987, Scripta Met., 21,1071

/16/ PATOOR E., 1986, "Contribution à l'étude de la plasticité de transformation dans les mono et polycristaux métalliques.", Thèse Université de Metz

/17/ MITTER W., 1987, "Umwandlungsplastizität und ihre Berücksichtigung bei der Berechnung von Eigenspannungen.", Gebruder Borntraeger, Berlin Stuttgart.

/18/ GIUSTI J., 1981, "Contraintes et déformations résiduelles d'origine thermique, application au soudage et à la trempe des aciers.", Thèse Université de Paris Vl

/19/ CLINARD F.W., SHERBY O.D., 1964, "Strength of iron during allotropic transformation.", Acta Met., 12, 911

/20/ KOT R.A., WEISS V., 1967, "Transformation Plasticity of Steels and Titanium in Torsion.", Trans. Am. Soc. Met., 60,566

/21/ SJÖSTRÖM S., 1984, "Interactions and Constitutive Models for the Calculation of Quench Stresses.", Proc. Int. Symp. on Calc. Int. Str. in Heat Treat. Met. Mat., Linkoping (Sweden)

122/ ABBES B., PILVIN P., 1993, "Identification de conditions aux limites en thermique pour la simulation de traitements thermiques.", 11 ème Congrès français de mécanique, Lille (à paraître)

123/ INOUE T., WANG Z.-G., MIYAO K., 1989, "Thermal and Phase Transformation Stresses in a Carburized-quenched Gear Wheel.", Proc. 32nd Japan Congress Mat. Research, Ed. The Society of Materials, Japan, 21-26 